
strains http://ow.ly/xDiM304ul7O

David Krieger ${ }^{1}$, Nicolas Schönfeld ${ }^{1}$, Silvan Vesenbeckh ${ }^{1}$, Gudrun Bettermann ${ }^{2}$, Torsten Thomas Bauer ${ }^{1,3}$, Holger Rüssmann ${ }^{2}$ and Harald Mauch ${ }^{2}$

${ }^{1}$ Klinik für Pneumologie, Lungenklinik Heckeshorn, HELIOS Klinikum Emil von Behring, Berlin, Germany. ${ }^{2}$ Institut für Mikrobiologie, Immunologie und Laboratoriumsmedizin, HELIOS Klinikum Emil von Behring, Berlin, Germany.

${ }^{3}$ Deutsches Zentralkomitee zur Bekämpfung der Tuberkulose (DZK), Berlin, Germany.

Correspondence: David Krieger, Klinik für Pneumologie, Lungenklinik Heckeshorn, HELIOS Klinikum Emil von Behring, Walterhoeferstr. 11, 14165 Berlin, Germany. E-mail: david.krieger@helios-kliniken.de

Received: July 152016 | Accepted after revision: Sept 132016 | First published online: Nov 112016

Conflict of interest: None declared.

\title{
References
}

1 Matsumoto M, Hashizume H, Tomishige T, et al. OPC-67683, a nitro-dihydro-imidazooxazole derivative with promising action against tuberculosis in vitro and in mice. PLoS Med 2006; 3: e466.

2 Skripconoka V, Danilovits M, Pehme L, et al. Delamanid improves outcomes and reduces mortalitiy in multidrug-resistant tuberculosis. Eur Respir J 2013; 41: 1393-1400.

3 Sotgiu G, Pontali E, Centis R, et al. Delamanid (OPC-67683) for treatment of multi-drug-resistant tuberculosis. Expert Rev Anti Infect Ther 2015; 13: 305-315.

4 Tadolini M, Garcia-Prats AJ, D'Ambrosio L, et al. Compassionate use of new drugs in children and adolescents with multidrug-resistant and extensively drug-resistant tuberculosis: early experiences and challenges. Eur Respir J 2016; 48: 938-943.

5 Tadolini M, Lingtsang RD, Tiberi S, et al. First case of extensively drug-resistant tuberculosis treated with both delamanid and bedaquiline. Eur Respir J 2016; 48: 935-938.

6 Diacon AH, Dawson R, Hanekom M, et al. Early bactericidal activity of delamanid (OPC-67683) in smear-positive pulmonary tuberculosis patients. Int J Tuberc Lung Dis 2011; 15: 949-954.

7 Xu HB, Jiang RH, Li L. Treatment outcomes for Mycobacterium avium complex: a systematic review and meta-analysis. Eur J Clin Microbiol Infect Dis 2014; 33: 347-358.

8 Griffith DE, Aksamit T, Brown-Elliot BA, et al. An official ATS/IDSA statement: diagnosis, treatment, and prevention of nontuberculous mycobacterial diseases. Am J Respir Crit Care Med 2007; 175: 367-416.

9 Schönfeld N, Haas W, Richter E, et al. Recommendations for diagnosis and treatment of nontuberculous mycobacterioses of the German Central Committee against tuberculosis and the German Respiratory Society. Pneumologie 2013; 67: 605-633.

10 Raju RM, Raju SM, Zhao Y, et al. Leveraging advances in tuberculosis diagnosis and treatment to address nontuberculous mycobacterial disease. Emerg Infect Dis 2016; 22: 365-369.

11 Pontali E, Sotgiu G, D'Ambrosio L, et al. Bedaquiline and multidrug-resistant tuberculosis: a systematic and critical analysis of the evidence. Eur Respir J 2016; 47: 394-402.

12 Radenbach KL. Diagnostische und therapeutische Fortschritte bei nichttuberkulösen Mykobakteriosen [Diagnostic and therapeutic progress in nontuberculous mycobacterioses]. Prax Klin Pneumol 1985; 39: 43-49.

13 Schönfeld N, Bergmann T, Vesenbeckh S, et al. Minimal inhibitory concentrations of first-line drugs of multidrug-resistant tuberculosis isolates. Lung India 2012; 29: 309-312.

14 Kent PT, Kubica GP. Public health mycobacteriology: a guide for the level III laboratory. Atlanta, USDHHS, Centers for Disease Control, 1985.

15 McClatchy JK. Antimycobacterial drugs: mechanism of action, drug resistance, suscebtibilitiy testing and assays of activity in biological fluids. In: Lorian V, ed., Antibiotics in Laboratory Medicine, 2nd Edn. Baltimore, The Williams and Wilkins Co., 1986; pp. 181-222.

\section{Specific airway resistance in preschool children: why not panting after all?}

\author{
To the Editor:
}

Specific airway resistance (sRaw) is measured with minimal cooperation in the preschool child during tidal breathing [1]. Methodological difficulties have been encountered in modern plethysmographs when the warming and humidification of the inspired gas [2] are replaced by numerical algorithms to eliminate the thermo hygrometric artefact [1,3,4]. Measuring sRaw during panting [5] had been dismissed in preschool children based on the assumption that the ventilatory manoeuvre would be difficult to perform and 
standardise. The feasibility of such an assumption, however, has not been verified. Therefore, the aim of this study was to assess the feasibility of measuring sRaw during panting in preschool children with asthma and compare the outcome with the tidal breathing method.

Preschool children with a doctor diagnosis of asthma were recruited from the paediatric pulmonology clinics (Hôpital d'enfants, Vandoeuvre-lès-Nancy, France). Written informed consent was obtained from the children and their parents. The protocol was approved by the ethics committee (Comité de Protection des Personnes EST III, CHU de Nancy, Nancy, France). The plethysmography equipment, which has been described elsewhere [6], is operated by software that includes an algorithm which should eliminate the thermo hygrometric artefact from the plethysmographic volume signal $\left(\Delta \mathrm{V}_{\text {pleth }}\right)$, which may be activated during tidal breathing and disabled during panting. Acquisition consisted of a series of four breaths selected automatically by the software and displayed on the computer screen. Three acquisitions were collected for each condition. Measurements were selected to retain those showing no artefact and where breathing frequency was lower than 50 breaths $\min ^{-1}$ during tidal breathing or higher than 60 breaths per. $\mathrm{min}^{-1}$ during panting. sRaw was computed between points of maximum $\Delta$ V pleth (sRtot) and in the flow range $\pm 0.5 \mathrm{~L} \cdot \mathrm{s}^{-1}\left(\mathrm{~s} R \mathrm{aw}_{0.5}\right)$. The overall flow interval within a given acquisition was measured graphically. Data were analysed with Pearson's Chi-squared test or analysis of variance as appropriate, and are expressed as mean $\pm \mathrm{SD}$.

A total of 127 preschool children (73 boys) aged 3.5-6.5 years took part in the protocol. Measurements were achieved during tidal breathing in 83 children $\left(34 \pm 7\right.$ breaths $\cdot \mathrm{min}^{-1}$; flow amplitude $1.3 \pm 0.3 \mathrm{~L} \cdot \mathrm{s}^{-1}$ ) and during panting in 90 children $\left(130 \pm 36\right.$ breaths $\cdot \mathrm{min}^{-1}$; flow amplitude $2.0 \pm 0.6 \mathrm{~L} \cdot \mathrm{s}^{-1} ; \mathrm{p}<0.0001$ for both breathing frequency and flow amplitude). Feasibility of measurement during tidal breathing and panting was not significantly different overall (65 and $71 \%$ respectively; $\mathrm{p}=0.35$ ), nor within age categories (figure 1a; $\mathrm{p}>0.05$ ). There was no difference between boys and girls during tidal breathing (64 versus $67 \%$; $\mathrm{p}=0.9$ ) or panting ( 71 versus $70 \% ; \mathrm{p}=0.9$ ). In those with successful measurements, the average number of validated breaths was $8.9 \pm 2.8$ during tidal breathing and 9.6 \pm 2.4 during panting $(\mathrm{p}=0.089)$. The percentage of valid breaths obtained did not differ between either breathing regimens (figure 1b) or sexes.

$\mathrm{s} R \mathrm{aw}_{0.5}$ and $\mathrm{s} R$ tot were significantly smaller during panting $\left(0.71 \pm 0.2 \mathrm{kPa} \cdot \mathrm{s} \cdot \mathrm{L}^{-1}\right.$ and $\left.0.96 \pm 0.3 \mathrm{kPa} \cdot \mathrm{s} \cdot \mathrm{L}^{-1}\right)$ than the respective value during tidal breathing $\left(1.14 \pm 0.2 \mathrm{kPa} \cdot \mathrm{s} \cdot \mathrm{L}^{-1}\right.$ and $1.33 \pm 0.2 \mathrm{kPa} \cdot \mathrm{s} \cdot \mathrm{L}^{-1} ; \mathrm{p}<0.0001$ for both $\mathrm{s} R \mathrm{aw}_{0.5}$ and $\mathrm{s} R$ tot). $\mathrm{s} R$ tot was also significantly larger than $\mathrm{s} R \mathrm{aw}_{0.5}$ for both protocols $(\mathrm{p}<0.0001)$. The intra-subject coefficient of variation was significantly larger during panting than during tidal breathing for both $\mathrm{sRaw}_{0.5}(13 \pm 7 \%$ versus $8.5 \pm 6 \%$; $\mathrm{p}<0.0001)$ and $\mathrm{s} R$ tot $(15 \pm 9 \%$ versus $10 \pm 7 \%$; $\mathrm{p}=0.0001)$ and significantly larger for $s R$ tot than $s R_{a} w_{0.5}$ during panting $(\mathrm{p}=0.02)$.

The potential of $s$ aw in paediatric lung function testing was formerly demonstrated in studies where the thermal component in $\Delta \mathrm{V}$ pleth was properly eliminated by warming and humidifying the respired gas [2]. Recent studies eventually showed important equipment-dependent variability [1,3] when numerical algorithms were used to correct for the thermo hygrometric artefact. The lack of agreement on sRaw standardisation in lung function centres across the world was recently highlighted [7]. The finding that panting achieves a similar rate of successful measurements as tidal breathing suggests the interesting possibility of avoiding the methodological difficulties encountered with automated software that corrects for the thermo hygrometric component using algorithms only known to the manufacturers. With smaller tidal volumes and higher breathing frequencies, the thermo hygrometric component in $\Delta \mathrm{V}_{\text {pleth }}$ is minimised [8] and the $\Delta V$ pleth-flow relationship is studied within a time domain mostly unaffected by the dynamics of airways - gas thermo hygrometric exchanges [9].
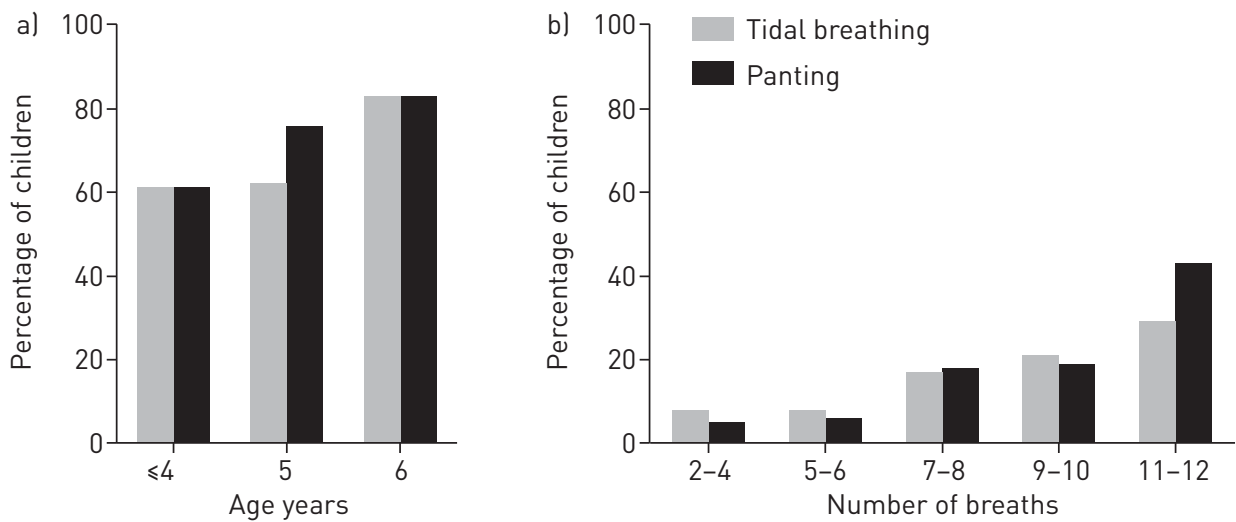

FIGURE 1 a) The success rate of specific airway resistance among age groups and b) the percentage of children achieving a given number of valid breaths are similar during tidal breathing and panting. 
The current results raise the question of which estimate of $s$ aw would be optimal as a routine paediatric lung function end-point. Panting opens the glottis [10], but turbulent airstream has the opposite influence on the measurement outcome. When $s R_{a w_{0.5}}$ is examined, however, the second mechanism is minimised, and the glottic opening should be more fully expressed to decrease sRaw. In fact, similar to a previous report in older children [6], in the current study sRaw was consistently lower during panting than during tidal breathing, and the highly significant difference was observed with both $s R$ tot and $s R_{a} w_{0.5}$. During tidal breathing, sRaw was also reported to be smaller in preschool children when the respired gas was conditioned to body temperature, ambient pressure, saturated with water vapour (BTPS) than when it was numerically corrected for the thermo hygrometric effect [11]. Taken together, these observations in preschool children indicate that either BTPS conditioning or panting, by adequately correcting for the thermo hygrometric artefact, lead to an estimate of sRaw being smaller than that achieved through software corrections applied during tidal breathing. However, the intra-subject variability in the current study was larger during panting than during tidal breathing, and whatever the mechanism, this might impede the ability of sRaw to detect airway obstruction and reversibility. The fact that $s R$ tot was significantly larger than the corresponding $s R_{a w_{0.5}}$ was expected because of the enhanced contribution of nonlinearities and increased slope of airway pressure-flow relationship. Since flow is turbulent mostly in proximal airways, the diagnostic value of $s R$ tot may be lower than for $s R_{a w_{0.5}}$. Furthermore, the larger variability observed with $s R$ tot compared with $s R a w_{0.5}$ was also expected, especially during panting, as the ventilatory effort is likely to vary from one breath to another, resulting in variable flow amplitude and contribution of nonlinearities throughout the acquisition. Hence, computation over a limited flow interval as reported in adults should be recommended [12], with the recent indication of better separation of children with asthma from controls based on $\mathrm{sRa}_{0.5}$ [13]. However, it should be kept in mind that, in preschool children where $\Delta V$ pleth and flow signals are small, computation of sRaw within a limited flow range may compromise the signal-to-noise ratio. The fixed flow interval $\pm 0.5 \mathrm{~L} \cdot \mathrm{s}^{-1}$ is also likely to provide age-dependent estimates of sRaw with more significant nonlinearities in shorter than taller children. An important step towards standardisation would be to define the optimal flow range to estimate sRaw and the optimal breathing frequency interval for measuring with minimal variability.

In summary, valid measurements of sRaw may be obtained during panting in preschool children. Since $\Delta \mathrm{V}_{\text {pleth }}$ requires no correction, the test should be more readily applicable to any equipment, which is particularly helpful in the context of international collaborative studies. Compared with tidal breathing, however, the technique achieves lower sRaw and larger intra-subject variability. Further case-control studies, including other lung function outcomes such as spirometry, are required to establish normative data and assess which estimate of $s R a w$ is the most useful for routine paediatric lung function studies. http://ow.ly/pvxr3034Bo8

Iulia Ioan $^{1,2}$, Silvia Demoulin-Alexikova ${ }^{1,2}$, Laurianne Coutier ${ }^{1,2}$, Claude Bonabel ${ }^{1,2}$, Jane Kirkby ${ }^{3}$, Kim G. Nielsen ${ }^{4}$, Waldemar Tomalak ${ }^{5}$, Bruce Thompson ${ }^{6}$, Cyril Schweitzer ${ }^{1,2}$, Paul D. Robinson ${ }^{3,7,8}$ and François Marchal ${ }^{1,2}$

${ }^{1}$ Dept of Pediatric Lung Function Testing, Children Hospital, Vandoeuvre, France. ${ }^{2}$ Dept of Physiology, EA 3450, Faculty of Medicine, University of Lorraine, Vandoeuvre, France. ${ }^{3}$ University College London, Institute of Child Health, London, UK. ${ }^{4}$ Danish PCD \& chILD Centre, CF Centre Copenhagen, Paediatric Pulmonary Service, Dept of Paediatrics and Adolescent Medicine, Copenhagen University Hospital, Rigshospitalet, Copenhagen, Denmark. ${ }^{5}$ National Research Institute for Tuberculosis and Lung Diseases, Rabka Branch, Rabka, Poland. ${ }^{6}$ Physiology Service Allergy, Immunology and Respiratory Medicine, The Alfred Hospital, Melbourne, Australia. ${ }^{7}$ Dept of Respiratory Medicine, The Children's Hospital at Westmead, Sydney, Australia. ${ }^{8}$ Dept of Paediatrics and Child Health, University of Sydney, Sydney, Australia.

Correspondence: François Marchal, Service d'Explorations Fonctionnelles Pédiatriques, Hôpital d'enfants, rue du Morvan, 54511 Vandoeuvre, France. E-mail: f.marchal@chru-nancy.fr

Received: April 112016 | Accepted after revision: Aug 042016 | First published online: Oct 202016

Conflict of interest: None declared.

Acknowledgements: Waldemar Tomalak passed away while the study was progressing. The members of the working group miss a good friend and an esteemed collaborator. They address their sincere condolences to his family.

\section{References}

1 Kirkby J, Stanojevic S, Welsh L, et al. Reference equations for specific airway resistance in children: the Asthma UK initiative. Eur Respir J 2010; 36: 622-629.

2 Dab I, Alexander F. A simplified approach to the measurement of specific airway resistance. Pediatr Res 1976; 10: 998-999.

3 Poorisrisak P, Vrang C, Henriksen JM, et al. Accuracy of whole-body plethysmography requires biological calibration. Chest 2009; 135: 1476-1480. 
4 Paton J, Beardsmore C, Laverty A, et al. Discrepancies between pediatric laboratories in pulmonary function results from healthy children. Pediatr Pulmonol 2012; 47: 588-596.

5 Dubois AB. Airway resistance. Am J Respir Crit Care Med 2000; 162: 345-346

6 Coutier L, Varechova S, Demoulin B, et al. Specific airway resistance in children: panting or tidal breathing? Pediatr Pulmonol 2014; 49: 245-251.

7 Robinson PD, Stocks J, Marchal F, et al. Poor standardisation of plethysmographic specific airways resistance measurement despite widespread use. Eur Respir J 2015; 46: 1811-1814.

8 Dubois AB, Botelho SY, Comroe JH Jr. A new method for measuring airway resistance in man using a body plethysmograph: values in normal subjects and in patients with respiratory disease. J Clin Invest 1956; 35: 327-335.

9 Peslin R, Duvivier C, Vassiliou M, et al. Thermal artifacts in plethysmographic airway resistance measurements. J Appl Physiol 1995; 79: 1958-1965.

10 Stănescu DC, Clément J, Pattijn J, et al. Glottis opening and airway resistance. J Appl Physiol 1972; 32: 460-466.

11 Klug B, Bisgaard H. Measurement of the specific airway resistance by plethysmography in young children accompanied by an adult. Eur Respir J 1997; 10: 1599-1605.

12 Briscoe WA, Dubois AB. The relationship between airway resistance, airway conductance and lung volume in subjects of different age and body size. J Clin Invest 1958; 37: 1279-1285.

13 Coutier L, Ioan I, Sadegh-Eghbali A, et al. Flow dependence of specific airway resistance and diagnostic of asthma in children. Pediatr Pulmonol 2015; 50: 1107-1112.

\title{
Dichotomy in pulmonary graft-versus-host disease evident among allogeneic stem-cell transplant recipients undergoing lung transplantation
}

\author{
To the Editor:
}

Allogeneic haematopoietic stem-cell transplantation (HSCT) has become a life-saving treatment option for numerous benign and malignant diseases, with more than 14500 procedures being performed annually in Europe alone [1]. Late-onset noninfectious pulmonary complications (NIPCs) have emerged as the main hurdle to long-term survival, affecting up to $26 \%$ of HSCT recipients and conferring 2- and 5-year survival rates of $44 \%$ and $13 \%$, respectively $[2,3]$.

Current diagnostic criteria remain based on those proposed by the National Institutes of Health (NIH) in 2005 for pulmonary graft-versus-host disease (GvHD), which considered bronchiolitis obliterans exclusively accountable $[4,5]$. Whilst histological confirmation was encouraged, invasive diagnostics have remained contentious due to reported complication rates from open lung biopsies and limited interpretability of trans-bronchial biopsies [6,7].

Inevitably, surrogate parameters such as new-onset airway obstruction on spirometry and "air-trapping" on computed tomography (CT) have gained precedence in establishing a diagnosis. NIH guidance for monitoring pulmonary GvHD currently incorporates the lung function score that assesses symptoms, forced expiratory volume in $1 \mathrm{~s}$ (FEV1) and diffusing capacity of the lung for carbon monoxide. Currently, diagnosis of pulmonary GvHD requires [4]: 1) FEV1/forced vital capacity (FVC) $<0.7$, FEV $1<75 \%$ predicted or residual volume $>120 \%$ predicted; 2) high-resolution CT demonstrating air trapping, small airway thickening or bronchiectasis; 3) exclusion of infection; and 4) chronic GvHD in at least one extrapulmonary site.

Recently, evidence has emerged suggesting the presence of an additional restrictive form, due to interstitial disease rather than skin GvHD of the chest wall, as has been previously suggested $[8,9]$. Through novel assessment of explanted native lungs from patients undergoing lung transplantation (LTx) for end-stage lung disease following allogeneic stem cell transplantation, we compared clinical, radiographic and histological data in this sub-group in an attempt to improve understanding of the pathological processes involved.

Patients who underwent cadaveric LTx between May 1, 2003 and May 1, 2014 at 12 European centres were included. Clinical, imaging and pathological data were collected using standardised reporting forms to facilitate central retrospective analysis. Spirometry was performed in accordance with American Thoracic 